\title{
Targeted genome editing in primate embryos
}

\author{
Cell Research (2015) 25:767-768. doi:10.1038/cr.2015.64; published online 2 June 2015
}

\begin{abstract}
Mosaic mutations and off-target effects caused by CRISPR/Cas9 have led to concerns about the efficiency and specificity of this new technique in non-human primates and other large animals. Here we discuss recent findings from primate embryos, with a focus on the technical issues CRISPR/Cas9 faces before producing non-human primate models of human diseases.
\end{abstract}

In the CRISPR/Cas9 (clustered regularly interspaced short palindromic repeats) system, specifically designed guide RNA (gRNA) directs nuclease Cas9 to the genomic DNA in a sequence-specific manner, and Cas9 cuts both strands at a precise location. The genomic DNA is then repaired by non-homologous end joining (NHEJ) or homologous recombination (HR), resulting in mutations that interrupt the open reading frame and cause gene inactivation. Because CRISPR/Cas9 allows the genome to be cut at virtually any location [1], today the system has become a powerful tool to genetically modify genomes in a variety of species, including human embryos [2] and nonhuman primates [3-5].

The development of CRISPR/Cas9 for genomic editing offers us an elegant new way to generate large animal models of genetic disorders without the need to establish embryonic stem cells for genomic manipulation. To explore whether CRISPR/Cas9 can edit genomic DNA at the one-cell stage in non-human primates, several groups applied CRISPR/Cas9 to fertilized oocytes from monkeys and found that newborn monkeys successfully carry mutations at the targeted genes [3-5].
However, these studies revealed that CRISPR/Cas9 creates mosaic mutations in genomic DNA in non-human primate embryos, which was also seen recently in human nonviable embryos derived from human tripronuclear zygotes injected with CRISPR/Cas9 [2]. Because there are concerns about the safety of using CRISPR/Cas9 in human embryos and because there are also the serious ethical issues involved in human germline modification, it is important to investigate how we can use CRISPR/ Cas9 in non-human primate embryos to generate animal models that can faithfully mimic human diseases.

The first important issue with CRIS$\mathrm{PR} / \mathrm{Cas} 9$ is its off-target effects. In the CRISPR/Cas9 system, a guide RNA hybridizes a 20-nt DNA sequence immediately preceding an NGG motif (protospacer-associated motif or PAM), resulting in a double-strand break (DSB) 3 bp upstream of the NGG. Because this targeting relies on a few base pair matches, CRISPR/Cas9 may generate a number of nonspecific mutations across the billions of base pairs of the genome in a single cell. Such nonspecific mutations can be diluted over generations in small animals, whose breeding time is short. However, for large animals like monkeys, their sexual maturation usually requires 4-5 years; thus the offtarget issue is critical and can confound the phenotypes of founder animals.

Most studies of nuclease off-target effects have been performed in cultured human cells; however, whole-genome sequencing of human pluripotent stem cell (hPSC) clones revealed very few off-target mutations attributable to the nucleases [6]. It is important to deter- mine the off-target rate in the embryos of primates. Using CRISPR/Cas9 to target three monkey genes simultaneously, Niu et al. [3] found no evidence for off-target effects. Targeting the monkey P53 gene via CRISPR/Cas9 also did not generate any off-target events [4]. Similarly, Chen et al. [5] examined the top 13 off-target candidate genes and found no evidence for off-target effects in newborn monkey tissues in which the dystrophin gene was targeted by CRISPR/Cas9. On the other hand, human tripronuclear zygotes injected with CRISPR/Cas9 did show an off-target event; however, this appeared to be minimal, as whole-exome sequencing revealed the presence of only two offtarget indels in 6 Cas9-injected human embryos [2]. Extra sperm nuclear DNA in tripronuclear zygotes could increase off-target events in these abnormal human embryos. Given that most reports show minimal to no off-target effects of CRISPR/Cas9 in primate embryos, it is likely that the off-target effects can be prevented by designing highly selective gRNA containing adequate mismatched base pairs with other genes.

Another important issue with CRIS$\mathrm{PR} / \mathrm{Cas} 9$ is the mosaic mutations. The mosaicism problem may result from the prolonged expression of Cas9 mRNA. However, direct injection of Cas9 protein rather than Cas9 mRNA into cells also leads to mosaic mutations $[7,8]$. Alternatively, differential DNA repair and non-homologous recombination activities in zygotes and divided embryonic cells can also influence genetic mutation rates and mosaicism. Mosaic mutations may affect generation of animal models of genetic human diseases, 
because a specific genetic mutation in a human disease often occurs at the one-cell stage before cell division, such that the same mutation is present ubiquitously in every individual cell. Despite the unknown mechanisms behind mosaicism, mosaic mutations can result in loss of function if they disrupt the expression of functional proteins. The evidence to support this comes from a Duchenne muscular dystrophy (DMD) monkey model, in which three different mutations in the dystrophin gene cause the loss of dystrophin in monkey muscle and muscle atrophy, as seen in DMD patients [5]. Thus, if mosaic mutations in primate embryos result in loss of function, the mutant animals may show pathology similar to that caused by a monogenic mutation in human diseases.

An advantage of CRISPR/Cas9 is that it can create mutations in two alleles to cause a null mutation in the founder animals. Such an advantage is particularly important for generating large animal models of diseases, as the extended time it takes to breed large animals does not allow us to quickly mate heterozygous mutant animals to generate homozygous mutants. In addition, since CRISPR/Cas9 can disrupt two alleles in female animals, the female animals should also show pathology due to the complete loss of function of the targeted gene, even if the disease gene is $\mathrm{X}$-linked. To ensure that two alleles are disrupted, multiple sites of the desired gene can be targeted by CRISPR/Cas9. In this regard, CRISPR/Cas9 provides a powerful tool for generating non-human primate or large animal models of human diseases that are caused by the loss of function of specific genes.

It should be noted that quantitative analysis of mosaic mutations in the targeted gene is necessary to assess the functional consequences. Earlier reports about CRISPR/Cas9 targeting efficiency in monkeys used subcloned DNA to analyze mutation rates [3, 4]. Chen et al. [5] recently used tissue ge- nomic DNA to assess mutation rates in the targeted dystrophin gene in rhesus monkey muscle and found that up to $87 \%$ of this gene could be disrupted. Newly developed whole-genome detection methods, such as deep sequencing and other high-throughput methods, will help off-target evaluation. However, these assays require genomic DNA amplification via PCR, which may yield some artificial results by PCR over-amplification with a preference for some genomic DNA. Also, most of the reports used the T7EN1 assay to assess the mutation rate of amplified genomic DNA, which could give rise to artificial results if T7EN1 enzymatic digestion is not controlled properly. Rigorous quantification of mosaicism in embryos from one-cell to multiple-cell embryos will be especially important if we are to understand the mechanisms for the mosaicism phenomenon.

CRISPR/Cas9 has also been used to generate knock-in mutations in various species; however, this knock-in rate is much lower than the random indel mutation rate because the knock-in requires the precise homologous recombination of the donor DNA. The rapidly developing technology has greatly improved the knock-in efficiency of CRISPR/ Cas9. For example, through the use of NHEJ inhibitors, the knock-in rate was significantly increased in mammalian cells $[9,10]$. Recently, direct nuclear delivery of Cas9 protein complex with chemically synthesized dual RNA was reported to generate knock-in mice carrying a functional cassette with up to $50 \%$ efficiency [11]. Overall, the newly developed CRISPR/Cas9 system holds great promise for use in non-human primates and large animals to generate knock-in models of human diseases or to modify specific genes.

Due to the considerable differences in anatomy, physiology, and genomics between rodents and primates, we now need to use non-human primates as complementary models to investigate disease pathogenesis [12]. The development of genomic editing via CRISPR/ Cas9 makes it possible to generate non-human primate or large animal models to more faithfully mimic human diseases; however, there are still some technical limitations for its use in nonhuman primates. Thanks to important insights and findings from small animals, which have been widely studied with genomic editing approaches, these technical limitations will likely be overcome soon, and there will be more non-human primate and large animal models to validate important findings from small animal models or to reveal unique pathology and phenotypes that do not occur in small animals.

\section{Xiangyu Guo ${ }^{1}$, Xiao-Jiang Li ${ }^{1,2}$}

${ }^{1}$ Institute of Genetics and Developmental Biology, Chinese Academy of Sciences, Beijing 100101, China; '2Department of Human Genetics, Emory University School of Medicine, Atlanta, GA 30322, USA

Correspondence: Xiao-Jiang Li

E-mail: xli2@emory.edu

\section{References}

1 Cong L, Ran FA, Cox D, et al. Science 2013; 339:819-823.

2 Liang P, Xu Y, Zhang X, et al. Protein Cell 2015; 6:363-372.

3 Niu Y, Shen B, Cui Y, et al. Cell 2014; 156:836-843.

4 Wan H, Feng C, Teng F, et al. Cell Res 2015; 25:258-261.

5 Chen Y, Zheng Y, Kang Y, et al. Hum Mol Genet 2015; 24:3764-3774.

6 Veres A, Gosis BS, Ding Q, et al. Cell Stem Cell 2014; 15:27-30.

7 Sung YH, Kim JM, Kim HT, et al. Genome Res 2014; 24:125-131.

8 Kim S, Kim D, Cho SW, et al. Genome Res 2014; 24:1012-1019.

9 Chu VT, Weber T, Wefers B, et al. Nat Biotechnol 2015; 33:543-548.

10 Maruyama T, Dougan SK, Truttmann MC, et al. Nat Biotechnol 2015; 33:538-542.

11 Aida T, Chiyo K, Usami T, et al. Genome Biol 2015; 16:87.

12 Belmonte JC, Callaway EM, Churchland P, et al. Neuron 2015; 86:617-631. 